Disponível em

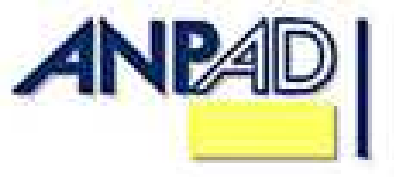

http://www.anpad.org.br/rac

RAC, Rio de Janeiro, v. 16, n. 6, art. 2,

pp. 784-805, Nov./Dez. 2012

(cc) EY-NG

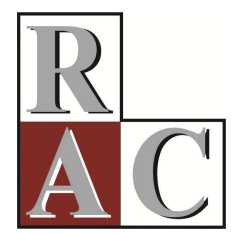

\title{
Conexões Políticas e Desempenho: um Estudo das Firmas Listadas na BM\&FBovespa
}

Political Connections and Performance: a Study of Firms Listed on the BM\&FBovespa

Sílvio Parodi Oliveira Camilo *

E-mail: parodi@parodiconsultoria.com.br Universidade Federal de Santa Catarina - UFSC

Florianópolis, SC, Brasil.

Rosilene Marcon

E-mail: rmarcon@univali.br

Universidade do Vale Itajaí - UNIVALI

Biguaçu, SC, Brasil.

Rodrigo Bandeira-de-Mello E-mail: rodrigo.bandeira.demello@fgv.br Fundação Getulio Vargas - EAESP-FGV São Paulo, SP, Brasil. 


\title{
Resumo
}

Esta pesquisa pretende contribuir para a disseminação dos estudos organizacionais que aliam governança e desempenho das corporações. Integra a perspectiva da dependência de recursos para explicar as conexões políticas das firmas na sua relação com o ambiente. Nesse contexto, foram investigadas três proxies que expressam ações políticas influentes no desempenho doação à campanha política; contratação de membros com background em política para compor a administração (diretoria e conselho); e atividades de board interlocking. $\mathrm{O}$ artigo apresenta um estudo longitudinal, estruturado de acordo com os mandatos presidenciais, compreendendo o período de análise de 1998 a 2009, composto por firmas não financeiras listadas na Bolsa de Valores de São Paulo (BM\&FBovespa). Para o teste de hipóteses, foram empregados o modelo multivariado de dados em painel e o método de efeitos fixos (fixed effects), considerando a firma e o tempo. Os resultados apontam que a conectividade com o ambiente político importa em aumento de valor da firma, medidos pelo Q de Tobin (modelos 1 e 2) e Market-to-book (modelos 3 e 4). As firmas fazem uso mais intenso dessas ações em períodos de maior incerteza política. Tais ações políticas são percebidas pelo mercado e refletidas nesses indicadores, que estão associados ao risco e à incerteza.

Palavras-chave: conexões políticas da firma; teoria da dependência de recursos; mecanismos de governança; conselho de administração.

\begin{abstract}
This research aims to contribute to the spread of organizational studies that combine performance and corporate governance. It utilizes the perspective of resource dependence to explain the political connections of firms as part of their relationships with their environments. In this context, we studied three proxies expressing political actions that influence performance: political campaign donations, recruiting members with backgrounds in politics to compose corporate administration (management and board), and interlocking activities between directors. The article presents a longitudinal study, structured according to presidential terms, for analysis of the period 1998 to 2009 of a sample composed of non-financial firms listed on the Stock Exchange of São Paulo (BM\&FBovespa). To test the hypothesis, we used multivariate modeling of panel data and the fixed effects method, which considered the firms and time. The results show that political connections increase firm value, as measured by Tobin's Q (Models 1 and 2) and Market-to-book ratio (models 3 and 4). Firms make greater use of political connections in periods of increased political uncertainty. Such policy actions are perceived by the market and reflected in indicators, which are associated with risk and uncertainty.
\end{abstract}

Key words: firm's political connections; resource dependence theory; governance mechanisms; board of directors. 


\section{Introdução}

A interação firma-ambiente é fundamental para a concepção de estratégias que capturem recursos vitais para a obtenção de melhores desempenhos (Hillman, 1999). Firmas buscam estreitar certos vínculos com o ambiente para extrair e preservar recursos essenciais ao seu valor (Agrawal \& Knoeber, 2001; Fisman, 2001; Hillman \& Keim, 1995; S. Johnson \& Mitton, 2003). A interdependência da organização com o ambiente afeta sua autonomia e estabelece incerteza em relação ao futuro, requerendo necessidades distintas e diferentes estratégias entre as firmas (Bazerman \& Schoorman, 1983; Pfeffer, 1972; Pfeffer \& Salancik, 2003).

De um lado, empresas que necessitem de financiamento para o equacionamento de dívidas ou para o funcionamento e o crescimento dos negócios, ou que atuem em indústria com regulação, podem intensificar suas estratégias políticas. De outro, o governo, como protagonista, exerce importante papel na economia, seja controlando e provendo recursos financeiros, deliberando políticas públicas, seja conduzindo organizações e atuando com proprietário de organizações. O arcabouço teórico aponta para a importância das relações políticas entre a firma e o poder público (Keim \& Baysinger, 1988; Mahon \& Murray, 1981; Pittman, 1977; Yoffie, 1988).

Considerando que as ações políticas das firmas são principalmente instrumentalizadas pela estrutura de propriedade e pelo conselho de administração, por mecanismos internos de governança, torna-se importante investigar as implicações das decisões dessas estruturas e o papel político influente no desempenho. Há um corpo teórico que investiga as ligações sociais das firmas por intermédio dos administradores, entendidos como membros do conselho de administração e executivos. Podem ser citados os estudos de J. Johnson, Daily e Ellstrand (1996), Westphal (1999) e Lynall, Golden e Hillman (2003). Mas a literatura sobre a governança política das firmas e os efeitos no desempenho, embora reúna uma série de trabalhos sobre o tema, reivindica novas contribuições. Abordagens que associam a dinâmica das firmas à política também vêm recebendo destaque.

O conselho de administração tem se mostrado atuante ao ampliar suas atividades, incorporando as de representação externa, influenciando, por sua vez, no desempenho (Bazerman \& Schoorman, 1983; Boyd, 1990; Brennan, 2006; Zahra \& Pearce, 1989). Considerando as relações políticas, a teoria da dependência de recursos enfatiza a importância de relacionar empresas com as contingências externas como forma de atenuar a incerteza e a interdependência entre a firma e o governo. Nessa direção, Hillman (2005) concluiu que empresas com políticos no conselho estão mais bem associadas a um melhor desempenho. Dentre as funções do conselho de administração, é do interesse deste estudo investigar os relacionamentos políticos como meio de aproximação que identificam os laços entre firma e governo, e analisar as implicações na performance das firmas.

Estudos sobre as conexões políticas das firmas são relativamente recentes. A literatura vem identificando certos benefícios mútuos dessa relação firma-governo, traduzidos em informação, influência, proteção, recursos financeiros e rendas do governo, demonstrando que há relação significativa entre a dependência de recursos do governo e a intensidade de conexões políticas (Fisman, 2001; Hillman \& Keim, 1995; Mahon \& Murray, 1981; Pittman, 1977). Embora os estudos empíricos tenham encontrado efeitos positivos no desempenho das firmas, é importante considerar o contexto político distintivo de cada país.

Dentre os estudos que abordaram as relações políticas entre firma e governo no Brasil, podem ser citados os de Samuels (2001a, 2001b, 2001c), Silva e Graminho (2005), Bandeira-de-Mello e Marcon (2005), Bandeira-de-Mello, Marcon e Alberton (2007), Ramalho (2007) e Claessens, Feijen e Laeven (2008). Concluiu-se que as empresas alimentam a expectativa de obter certos resultados, representados por devolução de favores, prestação de serviços, contratos, informações e outras vantagens (Samuels, 2001a, 2001b, 2001c). Essa aproximação pode se estabelecer por doação à campanha política, por meio da contratação de ex-políticos, para atuarem nos conselhos e na diretoria, e fortalecer-se pelas relações que esses membros possuem e desenvolvem ao participarem de outras organizações. 
Alinhando-se às recomendações da corrente que pesquisa a relação firma-governo, sob o âmbito da governança e do desempenho, este trabalho, com base na teoria da dependência de recursos, busca contribuir para explicar as diferenças no desempenho das firmas no contexto Brasil, respondendo a seguinte questão: as conexões políticas entre firma e governo, por doação à campanha, por antecedentes políticos da administração e interlocking do conselho de administração, têm influência no desempenho das empresas listadas na BM\&FBovespa?

$\mathrm{O}$ artigo analisa as conexões políticas das firmas brasileiras listadas na BM\&FBovespa, não financeiras e sem regulação, e suas influências no desempenho de mercado no período de 1998 a 2009. Cabe salientar que investigações sobre governança e desempenho que abordem conexões políticas das firmas com essa dimensão não foram encontradas até o momento no Brasil, o que representa uma provocação para quem pretende explorar esse aspecto.

\section{Revisão da Literatura}

\section{Mecanismos internos de governança}

Os mecanismos de governança corporativa (GC) são instituições econômicas e legais que podem ser modificadas por políticas de processos, algumas vezes para melhor (Shleifer \& Vishny, 1997). Os mecanismos de controle visam assegurar a proteção aos acionistas e evitar ou minimizar possíveis conflitos de interesses. Denis e McConnell (2003) classificaram os mecanismos de governança em internos (conselho de administração e estrutura de propriedade) e externos (mercado de aquisições e sistema legal).

Os membros do conselho de administração são eleitos pelos acionistas, a eles se subordinam e prestam contas e, por sua vez, indicam os diretores (agentes), monitoram suas atividades e controlam a geração de valor (Agrawal \& Knoeber, 1996; Zahra \& Pearce, 1989). Fazem parte da administração da firma o conselho de administração e a diretoria; todavia, somente os diretores exercem a representação da companhia (conforme estabelecem os Arts. 138 e 139 da lei no 6.404, 1976). Os mecanismos externos de GC, como o mercado, o sistema legal e regulatório e as instituições, agem no sentido de promover o equilíbrio das relações entre interesses dos acionistas, da firma e dos agentes (Denis \& McConnell, 2003; Rediker \& Seth, 1995).

\section{Estruturas de propriedade e controle}

Estudos que associam a estrutura de propriedade e controle ao desempenho vêm se mostrando frequentes na literatura. Como sugeriram Bohren e Odegaard (2004), além das variáveis e formulações usuais, outras variações e aproximações podem ser consideradas. O alinhamento de interesses entre os acionistas (controladores e minoritários), o conselho de administração e os gestores se mostra importante fonte geradora de desempenhos satisfatórios. A estrutura de propriedade e controle é um mecanismo determinante de resultados. Conforme as categorias em que se enquadram os acionistas controladores há variações nos mesmos e, por consequência, no valor da firma (Bohren \& Odeggard, 2004; Cho, 1998; Demsetz \& Lehn, 1985; Shleifer \& Vishny, 1986; Thomsen \& Pedersen, 2000).

A partir da investigação de Demsetz e Lehn (1985) sobre a concentração de propriedade e a maximização de valor de um conjunto de firmas, vários estudos foram elaborados. Dentre os quais, o trabalho de Thomsen e Pedersen (2000) que, ao examinarem o impacto da estrutura de capital no desempenho das firmas europeias, classificaram os acionistas em cinco categorias: bancos, investidor institucional, companhias não financeiras, indivíduos/família e governo. Nesse estudo, concluíram que as diferenças de relacionamento entre a concentração de voto das categorias são influentes no desempenho e na estratégia das corporações. Na mesma linha, Bohren e Odegaard (2004), ao analisarem as formas de interação e causação entre governança e desempenho, distinguiram os investidores em cinco categorias: governo, indivíduos, instituições financeiras, não financeiras e 
internacionais. Ao discorrerem sobre essa relação, entenderam que, devido à jovialidade da teoria da governança, as inferências de seus estudos podem não ser conclusivas, sugerindo outras combinações de variáveis para qualificar o poder explicativo entre mecanismos de governança e desempenho.

\section{Conselho de administração}

O conselho de administração é um mecanismo interno da governança corporativa que foi objeto de análises e formulações de pesquisadores como Pfeffer (1972), Fama (1980), Fama e Jensen (1983), Zahra e Pearce (1989), Boyd (1990), Daily e Dalton (1994a, 1994b), Rediker e Seth (1995), Hart (1995), Hillman, Cannella e Paetzold (2000) e Denis e McConnell (2003). Nesse sentido, o trabalho seminal de Zahra e Pearce (1989), ao investigar as influências do conselho de administração no desempenho financeiro da corporação, abordou quatro perspectivas teóricas: a legalista, a classe hegemônica, a teoria da agência e a dependência de recursos. Dentre as concepções estudadas, são predominantes a da teoria da agência e a da dependência de recursos. Para a teoria da agência, o conselho deve exercer um papel interno de intenso monitoramento e de preservação de interesses dos acionistas. Já para a teoria de dependência de recursos, o conselho está mais vinculado ao ambiente externo, exercendo, portanto, um papel mais proativo e contributivo ao valor da firma.

O conselho de administração, ao cumprir a missão de equilibrar os interesses dos gestores e dos acionistas (Agrawal \& Knoeber, 1996), assume um papel que exige destacável competência. Suas relações com o ambiente externo, disputando recursos do interesse da firma, foram consideradas como fundamentais, dada a escassez de recursos e os competidores pertencentes à arena (Pfeffer, 1972). Além dos aspectos relacionados ao background dos membros da administração, o tamanho medido pelo número de conselheiros é uma proxy que pode indicar a efetividade do órgão (Boyd, 1990).

A presença de conselheiros externos está bem relacionada a alto desempenho, porque eles fornecem certos benefícios, como acesso a recursos e informações importantes, facilitam compromissos interfirmas e contribuem no estabelecimento de legitimação (Hillman, Zardkoohi, \& Bierman, 1999; Schuler, Rehbein, \& Cramer, 2002; Westphal, 1999). A seleção de membros-outsiders com ricos-recursos pode fornecer inestimáveis vínculos com o ambiente (Boyd, 1990).

\section{Teoria da dependência de recursos}

Relacionar a firma ao ambiente é o propósito do construto teórico da dependência de recursos. A redução da incerteza ambiental pode ser administrada pela empresa, empregando determinadas estratégias (Pfeffer, 1972). Sob essa ótica, a firma é entendida como um organismo ativo, capaz de responder às influências do ambiente externo com adoção de estratégias voluntaristas (Aldrich \& Pfeffer, 1976; Pfeffer, 1972).

Essa relação entre a firma e o ambiente é um processo de troca. Se as organizações não podem promover, por si próprias, os recursos necessários requeridos, então devem supri-los interagindo com o ambiente (Aldrich \& Pfeffer, 1976). Essa interação é uma forma de responder aos fatores da competitividade, capturando recursos vitais à obtenção de melhores desempenhos (Pearce, 1983). Os atores envolvidos na construção desses elos são os acionistas, os chief executive officers $(C E O)$ e membros do conselho de administração (Agrawal \& Knoeber, 2001; Bertrand, Kramarz, Schoar, \& Thesmar, 2006; Boubakri, Cosset, \& Saffar, 2008; Fan, Wong, \& Zhang, 2007; Goldman, Rocholl, \& So, 2009; Leuz \& Oberholzer-Gee, 2006; Li, Meng, Wang, \& Zhou, 2008).

\section{Conexões políticas da firma}

As conexões políticas podem desempenhar uma função importante nas economias maiores e mais importantes do mundo; por isso, tornam-se um recurso valioso para muitas firmas (Fisman, 2001). Como regra geral, a organização está politicamente conectada quando há ligações com o governo por meio dos controladores, executivos ou membros do board. A companhia está conectada com um político se um dos maiores acionistas ou o representante principal é um membro parlamentar, 
um ministro chefe de Estado, e tenha estreitado relacionamento com o alto escalão. Se um membro do conselho ocupou uma posição como senador, membro da câmara dos deputados, membro da administração, e tenha atuado como diretor de uma organização ligada ao governo ou tenha sido nomeado para qualquer função pública (Boubakri et al., 2008; Faccio, 2006; Fan et al., 2007; Goldman et al., 2009).

O intercâmbio entre conselhos também é um modo de estabelecer laços com o ambiente. A presença de profissionais que compartilham com outros conselhos (interlocking) é uma prática usual e data do início do capitalismo (Dooley, 1969). A maximização dessas relações empresariais por meio do interlocking promove certos benefícios às organizações, contribuindo na redução das incertezas ambientais (Bazerman \& Schoorman, 1983; Mizruchi \& Stearns, 1988; Schoorman, Bazermanm, \& Atkin, 1981). A troca de conhecimentos e expertise transmite uma imagem favorável ao mercado (Barringer \& Harrison, 2000; Schoorman et al., 1981).

Por outro lado, a prática de interlocking também pode ser usada como meio de cooptar recursos de um ambiente incerto e ampliar a representação externa, em razão de declínio econômico e financeiro da organização (Mizruchi \& Stearns, 1988). Nesse sentido, esses autores entendem que o entrelaçamento entre os conselhos favorece a obtenção de recursos. Há convergência teórica de que o compartilhamento produz benefícios, porque influencia positivamente nos resultados (Hillman et al., 1999). Essa corrente que aborda os relacionamentos por intercâmbio entre conselhos entende que tais vínculos políticos da organização são importantes. Neste estudo, importa a identificação do interlocking dos membros da administração com antecedentes em política e que participem em conselhos de outras firmas com a presença de membros com experiência no governo, seja na administração direta, indireta e/ou legislativa. No Brasil, estudos que se dedicam às relações firmagoverno ainda são recentes. Em exame dos dados das eleições de 1994 e 1998 no Brasil, concluiu-se que parte significativa dos recursos de campanha provém das organizações (Samuels, 2001b). Contribuições de campanha estão mais bem associadas aos interesses recíprocos, dos doadores e donatários. Isso demonstra que há relação direta entre os interesses das corporações e a potencialidade que os cargos políticos comportam. Claessens et al. (2008), em relação às campanhas políticas de 1998 e 2002, encontraram evidências robustas de que altas contribuições à campanha de candidatos a deputado federal no país, especialmente aos vencedores, estão associadas com alto retorno das ações no momento do anúncio. As firmas que contribuíram se alavancaram substancialmente em relação às demais e obtiveram mais acesso às linhas de financiamento.

Nesse aspecto, o estudo de Lazzarini (2011) aborda justamente esses laços estabelecidos por intermédio de complexos grupos empresarias e pirâmides societárias. Ao analisar a dinâmica da rede de propriedade no país, aponta para a intensificação da participação do governo na economia, seja por meio dos fundos de pensão ou do Banco Nacional de Desenvolvimento Econômico e Social (BNDES). Os achados revelam mútuo interesse entre a firma e o governo, com reflexos positivos às organizações e negativos ao mercado, como concentração monopolista.

\section{Hipóteses Concernentes às Conexões Políticas}

A teoria da dependência de recursos, ao relacionar a firma ao ambiente, atribui um importante papel externo ao conselho de administração, estreitando laços e objetivando reduzir a interdependência e a incerteza ambiental. Esse vínculo com o ambiente permite a redução da assimetria de informações perante o ambiente de competição. Conexões políticas empreendidas por membros do conselho de administração, com antecedentes políticos em governos e com ligações políticas, facilitam acesso a fontes financeiras, informacionais e privilégios na disputa e na preservação de recursos, de modo a contribuir nas decisões estratégicas e, por consequência, no desempenho das firmas (Claessens, Feijen, \& Laeven, 2008; Faccio, 2006; J. Johnson, Daily, \& Ellstrand, 1996). 
Essa proposição se baseia nos pressupostos teóricos da governança, mais especificamente naqueles que se associam à perspectiva da teoria da dependência de recursos, em que elos da firma ao ambiente são guiados por estratégias políticas que visam à obtenção de desempenho superior. Para a teoria da dependência de recursos, a articulação ao ambiente é uma função direta dos níveis e tipos de dependência que uma organização possui (Hillman, Cannela, \& Paetzold, 2000). Logo, os laços estabelecidos pelas firmas expressam o grau de articulação e de dependência em relação ao ambiente.

Em síntese, a abordagem da dependência de recursos se apega à premissa de que a incerteza ambiental pode ser minimizada com estratégias delineadas pelas firmas (Aldrich \& Pfeffer, 1976; Daily \& Dalton, 1994a, 1994b; Pfeffer, 1972). Estabelecer interesses mútuos entre firma e governo é uma estratégia política que promove benefícios (Bandeira-De-Mello \& Marcon, 2005; Hillman, 1999; Hillman \& Hitt, 1999; Hillman et al., 2000). Envolvidos com as discussões desse corpo teórico, Hillman, Zardkoohi e Bierman (1999) e Schuler, Rehbein e Cramer (2002) enfatizam que ligações com o governo geram valor à firma.

No âmbito da governança o conselho de administração é um mecanismo interno com atividades relacionadas à minimização da dependência externa e ao ganho de recursos (Pfeffer, 1972). Mutações na composição dos conselhos, ampliando a participação de membros outsiders, revelam estratégias voltadas à aproximação ao ambiente e à redução da incerteza (Pearce \& Zahra, 1992). É justamente um dos aspectos que a TDR discute, ou seja, a presença de conselheiros externos sinaliza que a firma direciona atenção para o gerenciamento ambiental da organização (Daily \& Dalton, 1994a, 1994b).

Este estudo argumenta que a destinação de recursos para doação à campanha política e a presença de conselheiros com background político pressupõe que a firma possui estratégias voltadas à redução da interdependência e à minimização de incertezas em relação ao ambiente. $\mathrm{O}$ estreitamento relacional via board interlocking é outra maneira de os membros do conselho fortalecerem e estabeleceram laços com ex-políticos que servem a determinadas companhias e, por meio dessa aproximação interorganizacional, de obterem informações privilegiadas (Barringer \& Harrison, 2000; Bazerman \& Schoorman, 1983; Koenig, Gogel, \& Sonquist, 1979; Schoorman et al., 1981).

Nesse sentido, partindo do pressuposto de que o governo tem recursos atrativos, a firma desenvolve estratégia política com fins de, também, obter e preservar influências sobre as políticas públicas (Hillman \& Hitt, 1999). Assim, vínculos políticos, mediados pelo conselho, influenciam o desempenho e se tornam importantes num ambiente de competição. As ligações por meio do interlocking são consideradas ações políticas que facilitam o acesso a instituições financeiras (J. Johnson et al., 1996), desenvolvendo, através das relações, mecanismos de extração de rendas e contribuições que protegem a firma dos competidores (Boubakri et al., 2008; Fan et al., 2007) e até mesmo permitem agir politicamente perante as instituições para restringir oportunidades de recursos às competidoras (Leuz \& Oberholzer-Gee, 2006).

Pesquisadores que estudam as conectividades das firmas apontam que os laços políticos corporativos podem ser traduzidos em aumento de valor. Esses elos são estabelecidos por doação à campanha política, participação de membros da administração com background em política e experiência obtida através de compartilhamento entre conselhos de administração de firmas com membros com antecedentes em política. $\mathrm{O}$ argumento deste estudo é que a integração dessas formas de conectividades é influente no valor. Com base na hipótese central H1, são testadas duas variáveis de valor.

H1: Existe um relacionamento significativo entre as conexões políticas, a partir de laços corporativos, como meio de redução da incerteza e o desempenho da firma.

Importante observar que o alinhamento de interesses entre os acionistas, o conselho de administração e os gestores é comprovadamente fonte geradora de resultados satisfatórios (Bohren \& Odeggard, 2004; Cho, 1998; Demsetz \& Lehn, 1985; Shleifer \& Vishny, 1986; Thomsen \& Pedersen, 2000). Considerando que o conselho de administração é eleito pelos acionistas, sua composição indica a atuação política da firma, ou seja, pressupõe que membros com background político podem sinalizar 
estratégias políticas mais ou menos intensas. Se estudos comprovam que a tipologia da propriedade e o controle influenciam no desempenho das organizações, se o conselho é eleito pelo controlador e se as firmas se conectam ao governo para obter benefícios, é de se supor que determinadas categorias proprietárias são influentes e mais bem conectadas politicamente que outras.

H2: Existe um relacionamento significativo entre as conexões políticas, por intermédio do conjunto de mecanismos internos (conselho de administração e estrutura de propriedade e controle), como meio da redução da incerteza, e o desempenho.

Objetivando responder a questão central de pesquisa e testar as hipóteses anteriormente formuladas, a seguir serão estabelecidos os indicadores de desempenho que compreendem a proposição.

\section{Métodos e Dados}

\section{População, fonte e tratamento dos dados}

A população é composta pelo conjunto de firmas ativas, inativas e não-financeiras, excluídas as reguladas (permissionárias e concessionárias de serviços públicos), das listadas na BM\&FBovespa no período de 1998 a 2009. As empresas reguladas foram desconsideradas porque impedidas de doar recursos financeiros às firmas (Lei Eleitoral $n^{\circ}$ 9. 504, 1997, art. 24). O estudo compreende três anos de eleições para os cargos de Presidente, Governador, Senador e Deputado Federal e Deputado Estadual/Distrital, em pleitos eleitorais em 1998, 2002 e 2006. Os dados das firmas se referem ao período de 1999 a 2009. A população resultante foi extraída pelo método não probabilístico, pois determinadas firmas foram excluídas, por exemplo: concordatárias, em recuperação judicial ou extrajudicial e em processo falimentar. No conjunto, as firmas, os modelos, foram testados com as empresas com e sem patrimônio líquido negativo. Como restrição adicional, integraram a base de dados somente as firmas com um conjunto de dados há pelo menos três exercícios sociais sucessivos. A Tabela 1 apresenta as fontes, objetivos e procedimentos utilizados na pesquisa.

Os dados das demonstrações contábeis foram coletados da base de dados Economática ${ }^{\circledR}$. O conjunto de dados não experimentais do período analisado compreendeu a busca no Tribunal Superior Eleitoral (TSE), na base Economática ${ }^{\circledR}$, na BM\&FBovespa e na Infoinvest ${ }^{\circledR}$. De acordo com o propósito da pesquisa, o período de abrangência se justifica por compreender o segundo mandato do presidente Fernando Henrique Cardoso (1999 a 2002), o primeiro (2003 a 2006) e parte expressiva do segundo período de mandato do presidente Luiz Inácio Lula da Silva (2007 a 2009).

Quanto à dimensão do tempo, o estudo é caracterizado por ser longitudinal, expressando uma série de 12 anos de observações. Fez uso da técnica econométrica em painel, caracterizada pelo exame da unidade de análise - firma - acompanhada ao longo do tempo. No caso em estudo, interessa analisar os efeitos dos eventos que caracterizem conexão de natureza política da firma, mediada pelo conselho de administração e executivos, para verificar a influência no desempenho. Trata-se, portanto, de uma pesquisa empírica, fundamentada em dados secundários, que procura identificar e analisar as relações entre esses fenômenos.

As métricas de desempenho, por firma, foram elaboradas de acordo com as médias dos períodos de mandato. Por exemplo, os dados foram agrupados em três períodos: (a) doação em 1998, variáveis das firmas de acordo com as médias dos anos 1999 a 2002; (b) doação em 2002, variáveis das firmas de acordo com as médias dos anos 2003 a 2006; e (c) doação em 2006, variáveis das firmas de acordo com as médias dos anos 2007 a 2009. O ano de 2010 não foi considerado no estudo em razão de os dados da população não estarem completos. A presença e o número de ex-políticos no conselho de administração e interlocking foram levantados por empresa e por ano, sendo totalizados de acordo com os três períodos de análise. 
Tabela 1

\section{Fontes, Objetivos Secundários e Procedimentos da Coleta de Dados de Conexão}

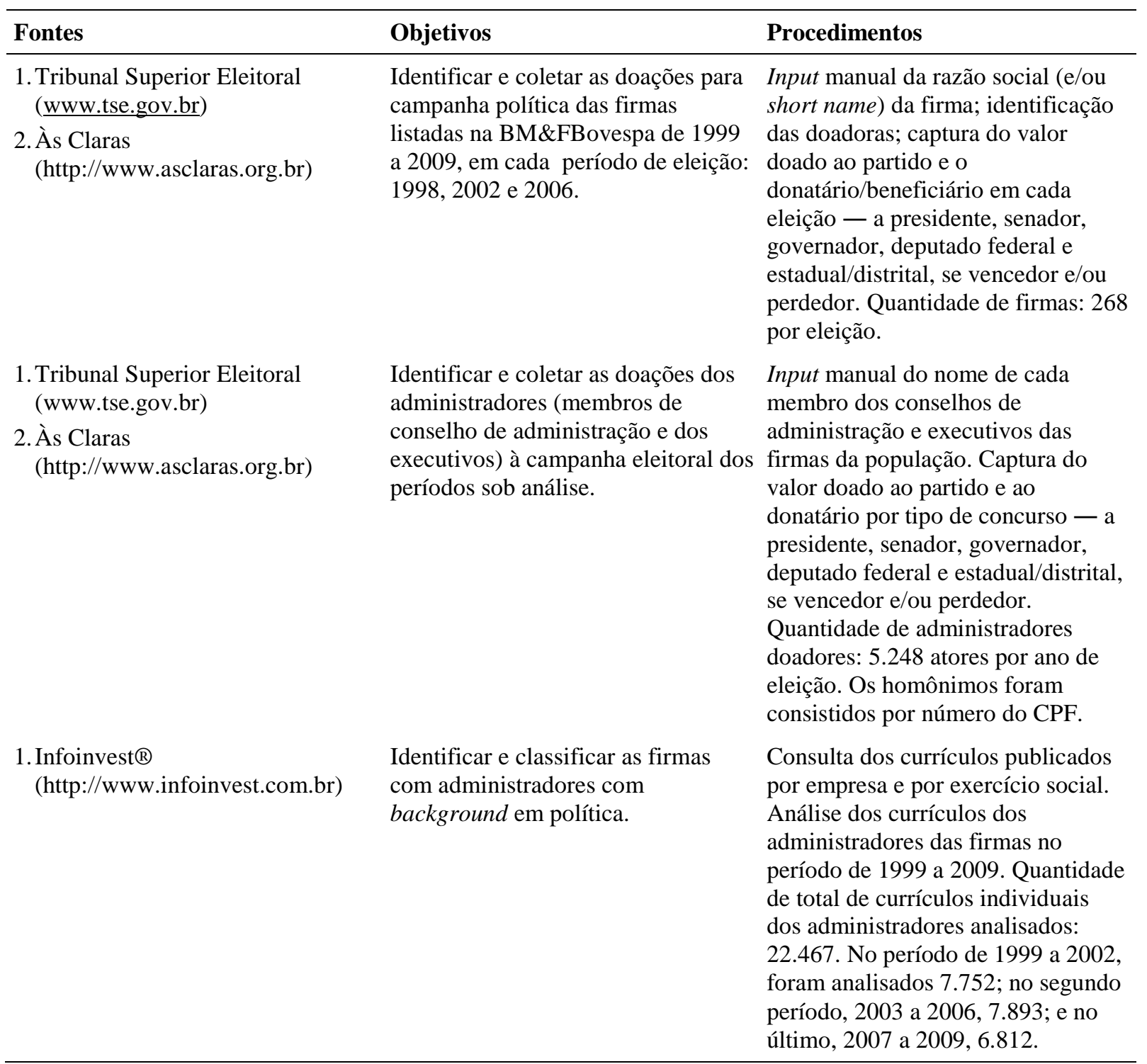

Nota. Fonte: Dados da pesquisa.

\section{Variáveis dependentes}

O desempenho da firma pode ser medido a partir de diversos níveis e bases conceituais, representando tema recorrente e central para a administração estratégica (Venkatraman \& Ramanujam, 1986). Visando testar as hipóteses elaboradas, foram testadas duas variáveis de desempenho de mercado: (a) valor da firma pelo Q de Tobin; e (b) valor da firma Market-to-book. O Q de Tobin ajustado é definido pela razão do valor de mercado das ações (ordinárias e preferenciais) mais a dívida contábil pelo valor contábil do ativo total (Chung \& Pruit, 1994; Jiang, 2008). O Market-to-book expressa o valor de mercado da firma, com base nas ações pelo valor do patrimônio líquido escritural (Fan et al., 2007; Jiang, 2008). 


\section{Variáveis independentes}

O conselho de administração é um importante elo interno e externo; daí a investigação das relações políticas da firma sob a ótica da dependência de recursos, considerando que práticas relacionais, ajustadas ao ambiente externo, têm por objetivo proporcionar benefícios (Bazerman \& Schoorman, 1983). Nesse sentido, na definição nas variáveis de ligações políticas das firmas, o estudo emprega as mensurações de conexões expostas na Tabela 2.

A literatura aponta que firmas que estreitam laços com o governo obtêm melhores resultados em relação às competidoras sem essas ligações. Com base nas atividades mapeadas por Brennan (2006), importa identificar a participação política do conselho no relacionamento com organismos externos.

Este estudo inova ao integrar a variável de interlocking de membros do conselho às métricas de conexões políticas das firmas, o que amplia os estudos empíricos até então conhecidos. Do compartilhamento entre os conselhos das firmas, importam somente as interações políticas. Nesse aspecto, interessa capturar as conexões políticas das firmas mediadas por conselheiros. Desse modo, por exemplo, as relações simultâneas com um ex-político de determinado conselho pressupõem a existência de estreitamento pessoal e compartilhamento de informações, incluídas as de cunho político.

Tabela 2

Variáveis Independentes de Conexão Política

\begin{tabular}{lll}
\hline $\begin{array}{l}\text { Variáveis de } \\
\text { aproximação }\end{array}$ & Descrição & Formas de Mensuração \\
\hline Doação & $\begin{array}{l}\text { O valor monetário doado ou os serviços } \\
\text { financiados declarados pela empresa nos } \\
\text { anos de eleições (1998; 2002; e 2006). }\end{array}$ & $\begin{array}{l}\text { Variável representativa do valor monetário } \\
\text { doado;/declarado; }\end{array}$ \\
$\begin{array}{l}\text { Background em } \\
\text { governo }\end{array}$ & $\begin{array}{l}\text { Com base no background do membro da } \\
\text { administração, identificação se é ou foi ex- } \\
\text { funcionário do governo ou político e quais } \\
\text { as experiências nessas atividades. }\end{array}$ & $\begin{array}{l}\text { (a) Variável representativa do número de } \\
\text { administradores com experiência em política } \\
\text { na Administração Direta; e (b) variável } \\
\text { representativa do número de administradores } \\
\text { com experiência no legislativo; }\end{array}$ \\
$\begin{array}{l}\text { Interlocking } \\
\text { político }\end{array}$ & $\begin{array}{l}\text { Com base na composição dos conselhos de } \\
\text { administração, identificação se há } \\
\text { compartilhamento político entre as firmas e } \\
\text { a quantidade de laços estabelecidos. }\end{array}$ & $\begin{array}{l}\text { Variável que expressa o número de } \\
\text { administradores com experiência política } \\
\text { compartilhada com outros conselhos que } \\
\text { possuem membros políticos (laços diretos). }\end{array}$ \\
\hline
\end{tabular}

Nota. Fonte: Dados da pesquisa.

Os laços diretos são decorrentes da presença de ex-políticos no conselho de administração da organização, de modo a expressar a conectividade com membros munidos de antecedentes políticos em outros conselhos. Também foram testados os laços indiretos interfirmas, inerentes ao compartilhamento entre membros do conselho sem antecedentes em política, de uma dada firma, com outros conselheiros, ex-políticos, de outras firmas. Esse interrelacionamento é analisado pela quantidade de nós estabelecidos a cada ano, ou seja, pelo número de compartilhamentos com conselheiros com esse conhecimento, agregados por período de mandato.

Em destaque, a utilização das variáveis binárias no modelo permitirá a segregação das firmas politicamente conectadas das sem conectividade, além da identificação das firmas com conexões mais intensas. A composição do conselho é determinada pelos acionistas da firma em assembleia geral ordinária (Lei $\left.\mathrm{n}^{\circ} 6.404,1976\right)$. Como é do interesse da pesquisa analisar os relacionamentos políticos das firmas, por meio da administração, e os efeitos no desempenho, torna-se fundamental compreender essas relações por categoria de estrutura de propriedade, ou seja, as como as categorias se associam aos modelos. Desse modo, o estudo segue a classificação sugerida por Faccio e Lang (2002) e Bohren 
e Odegaard (2004), ou seja, a origem do capital controlador diferenciado nas categorias de propriedade: indivíduos/família, instituições financeiras, organizações internacionais e corporações não financeiras.

$\mathrm{Na}$ identificação das categorias de identidade dos proprietários das firmas, utilizou-se uma variável binária (dummy), atribuindo-se 1 (um) para a associação da firma em determinada categoria e 0 (zero) para as categorias não associadas.

\section{Variáveis de controle}

As variáveis de controle são aquelas que possuem relacionamento com as variáveis dependentes do modelo (Q de Tobin, Market-to-book). Foram testadas as seguintes variáveis de controle: o porte da firma representado pelo ativo total (Fan et al., 2007; Titman \& Wessels, 1988), bem como a receita bruta (Titman \& Wessels, 1988). Em finanças, a variável tamanho de uma empresa é comumente medida com base no ativo total ou na receita bruta. Com base em Titman e Wessels (1988), no presente estudo adotou-se a receita bruta em razão da natureza das variáveis dependentes. Depois de recuperados os dados de origem da base Economática ${ }^{\circledR}$ e em razão de os dados não apresentarem normalidade, a receita bruta foi transformada, em segunda ordem, para o logaritmo natural (Titman \& Wessels, 1988).

Dado que a combinação ótima de fontes de financiamento importa no valor de mercado da empresa, outra variável de controle considerada no modelo foi o grau de endividamento. A escolha de fontes de financiamentos mais vantajosas produz melhores resultados à empresa, como a maximização de lucros aos proprietários, e a torna mais atrativa perante o mercado, porque influencia na escolha de potenciais investidores em ações (Abor, 2005; Myers, 1984; Myers \& Majiuf, 1984). Empresas alavancadas possuem menos restrições a fontes alternativas de capitais, estabelecendo certa influência no mercado, e devido a baixa assimetria informacional obtêm custos de transação mais favoráveis (Titman \& Wessls, 1988).

Com fins de minimizar as influências do tamanho dos conselhos, para fins de controle, utilizouse o logaritmo natural no número de membros do board. Esse critério está em conformidade com os estudos de Yermack (1996) e Forbes e Milliken (1999).

Para a melhor compreensão, a seguir será apresentada a especificação do modelo.

\section{Especificação do modelo empírico}

Conforme os modelos teóricos apresentados, a literatura sugere que os efeitos das conexões políticas são favoráveis à firma. Seguindo a proposta de pesquisa, o modelo elaborado examina os efeitos desses laços políticos no desempenho:

\section{Desempenho $=\mathbf{f}($ atributos da governança, conexões políticas, controle)}

$\mathrm{Na}$ especificação do modelo geral, o desempenho é função dos mecanismos internos de governança e das variáveis específicas da firma. Dentre os atributos da governança, são utilizados, no modelo, os mecanismos internos definidos pela identidade de propriedade e as atividades políticas do conselho de administração. As variáveis específicas são as de controle, que exercem influência nas variáveis dependentes.

O modelo é descrito da seguinte forma:

$$
\begin{gathered}
\text { Desempenhoi, } t=\mu 1+\beta 1 \text { doação i,t }+\beta 2 \text { background } i, t+\beta 3 \text { interlocking } i, t+\beta 4 \text { identidade de } \\
\text { propriedade } i, t+\beta \text { ThamFirma i,t }+\beta \text { Endividamento } i, t+\beta \text { TTamBoard } i, t+\alpha i+e i, t
\end{gathered}
$$

O desempenho da firma $i$, no período $t\left(1^{\circ}\right.$., $2^{\circ}$. e $\left.3^{\circ}\right)$, é definido pelas variáveis de dimensões de mercado (Q de Tobin e Market-to-book), $\mu_{I}$ representa o intercepto, as variáveis independentes são 
representadas pela doação, background, interlocking e identidade de propriedade. Além do endividamento, duas variáveis de controle foram consideradas, TamFirma e TamBoard, representadas pelo logaritmo natural da receita bruta e do número de membros do board, respectivamente. As variáveis $\alpha_{i}+\varepsilon_{i t}$ representam o efeito fixo, os desvios acumulados individuais em relação ao intercepto e a variação do erro observado em relação ao esperado no período $(t)$, respectivamente.

Em síntese, a estimação do modelo considera dados sobre o desempenho das firmas, doações à campanha política por cargo, presença de administradores com experiência em política como atributo de conectividade, interlocking dos membros de conselhos de administração com firmas cujos conselheiros possuem experiência política e categorias de propriedade e controle, mais variáveis de controle.

\section{Análises e Resultados}

\section{Abordagem analítica}

Diferentes estratégias de estimações podem ser usadas para análise em dados em painel. Petersen (2009) sugere, quando há poucos períodos e muitas observações em cada período, o uso de regressões com erros-padrão agrupados por firmas e com dummies para o controle dos efeitos do tempo. O agrupamento por firma permite relaxar a hipótese sobre a ausência de correlação serial, que é claramente violada em um conjunto de dados em painel com múltiplas observações para cada empresa (Okhmatovskiy, 2010). Com base no modelo em estudo, determinadas decisões de um período podem produzir efeitos em períodos posteriores ou, até mesmo, num momento imediato.

Visando capturar os efeitos das conexões políticas das firmas, foram inicialmente testados, no modelo multivariado de dados em painel, o método de efeitos fixos (fixed effects) e o de efeitos aleatórios (randon effects). Com base nos dois métodos, posteriormente foram realizados os testes de robustez. A estimação pelo método de efeitos fixos considera os efeitos não observáveis que podem estar associados à variável dependente. Nesse método, a variável dependente é mensurada numa escala de tempo e é linearmente dependente de um conjunto de variáveis preditoras (Wooldridge, 2003). No método de efeitos aleatórios, a estimação das variáveis explicativas não varia com o tempo. Pode-se supor que decisões de governança para estabelecer os laços firma-governo estejam vinculadas a determinadas características das firmas e relacionadas à melhoria de desempenho. Dessa forma, a natureza das variáveis explicativas aponta que as relações não são uma escolha aleatória e, além disso, variam com o tempo. Por essa razão, o método de efeitos fixos está mais bem relacionado à natureza desta pesquisa.

Com base nas hipóteses formuladas, para cada variável explicada, dois modelos de estimação foram aplicados. Na primeira etapa, foram testadas as variáveis de governança associadas a conselho de administração (modelos 1 e 3, hipótese H1). Num segundo estágio são incorporadas as variáveis de governança de propriedade e controle (modelo 2 e 4, hipótese H2).

\section{Resultados empíricos}

A Tabela 3 apresenta a estimação dos modelos (1) e (2), considerando a variável dependente Q de Tobin, e os modelos (3) e (4) referentes à variável Market-to-book. Essa tabela também dispõe dos coeficientes, da significância, do erro padrão e do $\mathrm{R}^{2}$ ajustado para as variáveis testadas correspondentes aos dados dos períodos em análise $\left(1^{\circ}, 2^{\circ}\right.$ e $\left.3^{\circ}\right)$. Pode-se destacar que, dos conjuntos de variáveis testadas, os que compõem a Tabela 3 foram os quais apresentaram significância estatística, além disso, indicaram se a variabilidade das variáveis Q de Tobin e Market-to-book pode ser explicada pelos modelos. 
Os resultados encontrados nessa tabela demonstram que os modelos estão adequados, pois $\mathrm{F}>0,05$, em que todos os coeficientes são diferentes de zero. Nesse caso, a soma dos quadrados explicados pelos modelos é relativamente maior do que a soma do quadrado dos resíduos, rejeitandose a hipótese de que o conjunto das variáveis explicativas não tem efeito sobre a variável explicada (Gujarati, 2006). Os coeficientes de determinação ajustados $\left(\mathrm{R}^{2}\right)$ dos modelos de efeitos fixos apontam que o conjunto de variáveis é capaz de explicar parte representativa da variabilidade da variável dependente.

O coeficiente de correlação intraclasse (rho), que resulta do quociente entre os desvios dos resíduos dentro das firmas e dos desvios totais, atesta a proporção da variabilidade, que é devida às diferenças entre firmas. As correlações entre os resíduos e os regressores nos modelos são muito baixas $(\operatorname{Corr}(u-i, x b))$.

As variáveis independentes de doação nos modelos (1) e (2) Q de Tobin, para presidente e governador, apresentam parâmetros com sinais contrários, porém ambos significativos, $p<0,05$. Nas firmas que doaram a candidato a presidente a relação foi inversa comparada ao valor esperado; já para o cargo de governador os parâmetros foram positivos. O trabalho também considerou as doações aos demais cargos, como para candidato a senado, deputado federal e deputado estadual/distrital, mas os resultados não foram significativos.

As variáveis de background em governo foram decorrentes das análises dos currículos dos administradores. Os dados considerados expressam a quantidade de habilidades dos administradores, traduzidas pelas experiências em governo, na administração direta e indireta, e no legislativo. No modelo (1), firmas com membros experimentados em política com precedente na administração direta não apresentaram parâmetro positivo, e a significância estatística foi $\mathrm{p}<0,10$. No modelo (2), essa variável não apresentou significância. Destaca-se que foram considerados e testados os dados das firmas com ex-políticos com antecedentes na administração indireta, mas não se mostraram relevantes. $\mathrm{O}$ interlocking entre firmas por intermédio de membros da administração, com experiência política, não apresentaram significância, em ambos os modelos.

Ainda considerando os modelos (1) e (2), ao se integrar a estrutura de propriedade, observa-se que as firmas com controle por indivíduos e/ou famílias apresentaram significância, $p<0,01$, com coeficientes positivos.

Com o objetivo de corrigir problemas de heteroscedasticidade, na regressão que tem como variável dependente o Q de Tobin, foram empregados testes estatísticos. Os resultados apontam para a estabilidade do modelo, pois não houve modificação substancial na significância, a ponto de comprometer o método de efeitos fixos, muito menos houve colinearidade. Destaca-se a intensificação da significância na doação a candidato a governador, de $\mathrm{p}<0,10$, para $\mathrm{p}<0,01$.

Conforme demonstra a Tabela 3, a equação Market-to-book foi estruturada com os mesmos grupos do conjunto de variáveis da métrica Q de Tobin, em ambos os modelos (1) e (2). Nos modelos (3) e (4), as variáveis de doação, tanto para os candidatos a presidente quanto ao governo estadual, foram significativas. Quanto aos sinais dos parâmetros, para o cargo a presidente, foi negativo, e para o cargo ao governo foi positivo.

Quanto às variáveis de background em governo, em ambos os modelos (3) e (4), firmas com membros com antecedentes políticos na administração direta não apresentaram parâmetro positivo e a significância estatística foi $\mathrm{p}<0,10$. Já em relação a firmas com membros ex-políticos com histórico curricular no legislativo, os parâmetros foram positivos, mas somente o modelo (3) apresentou significância. 
Tabela 3

Resultados da Regressão de Efeitos Fixos ${ }^{a}$

\begin{tabular}{lrrrr} 
& Q de Tobin & & Market-to-book & \\
\cline { 2 - 5 } & $(1)$ & $(2)$ & $(3)$ & $(4)$ \\
\hline Doação & & & & $-380 \mathrm{e}-07$ \\
Presidente & $-2,09 \mathrm{e}-07$ & $-1,88 \mathrm{e}-07$ & $-4,45 \mathrm{e}-07$ & $(0,083)+$ \\
& $(0,014)^{*}$ & $(0,027)^{*}$ & $(0,041)^{*}$ & $7,84 \mathrm{e}-07$ \\
Governador & $2,95 \mathrm{e}-07$ & $2,73 \mathrm{e}-07$ & $8,37 \mathrm{e}-07$ & $(0,013)^{*}$ \\
& $(0,015)^{*}$ & $(0,024)^{*}$ & $(0,007)^{* *}$ & $-0,064$ \\
& & & & $(0,087)+$ \\
Background & $-0,027$ & $-0,022$ & $-0,072$ & 0,192 \\
Adm. direta & $(0,058)+$ & $(0,126)$ & $(0,052)+$ & $(0,108)$ \\
& 0,123 & 0,102 & 0,228 & \\
Legislativo & $(0,007)^{* *}$ & $(0,026)^{*}$ & $(0,055)+$ & \\
\end{tabular}

\section{Interlocking}

Laços diretos

\begin{tabular}{rrrr}
0,020 & 0,019 & 0,054 & 0,051 \\
$(0,122)$ & $(0,140)$ & $(0,100)+$ & $(0,127)$ \\
\hline
\end{tabular}

\section{Propr. e controle}

Indivíduos/famílias

$0,202 \quad 0,266$

$(0,007)^{* *}$

Inst. financeiras

$-0,130$

$-0,360$

$(0,249)$

$(0,241)$

Estrangeiras

$-0,0405$

$-0,332$

$(0,730)$

$(0,303)$

\section{Controle}

TamFirma

$0,070 \quad 0,061$

0,188

0,173

Endividamento

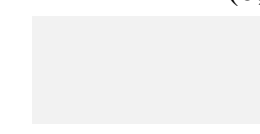

TamBoard

$\begin{array}{rr}-0,012 & -0,012 \\ (0,0)^{* * *} & (0,0)^{* * *}\end{array}$

$(0,052)+$

$(0,072)+$

1,059

$(0,086)+$

$(0,086)+$

$(0,0)^{* * *}$

$-0,022$

$-0,022$

\section{Efeitos}

Firma

$\begin{array}{rr}\text { Sim } & \text { Sim } \\ \text { Sim } & \text { Sim } \\ 478 & 478 \\ 232 & 232 \\ 0,721 & 0,729 \\ -0,102 & -0,106 \\ 0,000 & 0,000 \\ 7,239 & 12,227\end{array}$

$(0,0)^{* * *}$

$(0,0)^{* * * *}$

Período

$\mathrm{n}^{\circ}$ de observações

$\mathrm{n}^{\circ}$ firmas

Adj R-squared

Co-Rr $(u-i, x b)$

Prob $>\mathrm{F}$

$\mathrm{F}$

7,239

\begin{tabular}{rr} 
Sim & Sim \\
Sim & Sim \\
458 & 458 \\
228 & 228 \\
0,603 & 0,607 \\
$-0,126$ & $-0,122$ \\
0,000 & 0,000 \\
8,227 & 11,219 \\
\hline
\end{tabular}

Sim

im

458

228

607

0,122

11,219

Nota. A variável categoria de propriedade ficou fora do modelo, servindo de base para as demais categorias, devido a sua predominância.

Significativo a *** p $<0,001, * * \mathrm{p}<0,01, * \mathrm{p}<0,05 \mathrm{e}+\mathrm{p}<0,10$

a (erro padrão entre parênteses). 
O interlocking entre firmas por intermédio de membros da administração com experiência política apresentou significância em relação ao modelo (3). Nos modelos estudados, também se analisou a estabilidade do método escolhido, de efeitos fixos, processando-se o teste de robustez [vce(robust)]. Os resultados das regressões para os modelos analisados, (3) e (4), foram estáveis.

Considerando que o grau de endividamento pode influenciar no acesso a fontes de recursos, utilizou-se essa métrica como variável de controle somente nos modelos (3) e (4), Market-to-book. Em relação aos modelos Q de Tobin, o endividamento não foi considerado como controle, porque está contido na variável dependente e, uma vez mantido, poderia distorcer os resultados.

Visando capturar a eventual endogenia entre determinadas variáveis independentes, vários testes foram empregados. Por exemplo, pode-se se supor que haja uma relação estreita entre as doações à campanha política e a contratação de conselheiros com antecedência em política, ambos com influência direta sobre o desempenho. Nesse sentido, foi desenvolvido um modelo-teste com as variáveis citadas atuando como mediadoras e/ou moderadoras, diretamente às variáveis e ao modelo geral. Entretanto, os resultados não foram significativos.

Com objetivo, ainda, de dar mais robustez ao modelo geral, foram testadas outras variáveis de desempenho, tais como o retorno: sobre os capitais próprios (ROE), sobre os ativos (ROA) e operacional das vendas (RSV).

Também foram avaliados efeitos dessas ações na variação da taxa de crescimento da firma, medida pela variabilidade da receita operacional bruta. Entretanto, os resultados não apresentaram significância. Firmas com passivo a descoberto foram adicionadas à amostra, mas não houve alteração importante nos resultados.

\section{Discussão dos resultados}

O modelo geral foi construído a partir das hipóteses delineadas. Essas hipóteses foram elaboradas com base na literatura e testadas com objetivo de analisar se conexões políticas das firmas brasileiras listadas na BM\&FBovespa foram influentes no desempenho. A base teórica de sustentação aponta para a existência de relação positiva entre os laços políticos estabelecidos pelas firmas com o governo, demonstrando, assim, certo valor.

Conforme se depreende da revisão teórica, as atividades políticas das firmas são um meio de reduzir e minimizar risco da incerteza do ambiente (Aldrich \& Pfeffer, 1976; Daily \& Dalton, 1994a, 1994b; Pfeffer, 1972). A literatura aponta que essas estratégias produzem certos benefícios às firmas, como desempenho superior, informação, influência, proteção, acesso privilegiado a recursos financeiros e rendas provenientes do governo (Boubakri et al., 2008; Fan et al., 2007; Fisman, 2001; Keim \& Baysinger, 1988; Pittman, 1977; Yoffie, 1988).

Este estudo apresenta implicações teóricas e empíricas. Teóricas pela abrangência integrativa dos construtos modelados. Ao unir correntes que se debruçam sobre temas importantes como governança e desempenho, integra argumentos da teoria da dependência de recursos para contribuir na explicação das relações firma-ambiente, por intermédio das conexões políticas. Essa contribuição pode ser estendida e aprofundada em outros contextos. No campo empírico, este trabalho se afilia aos estudos que analisam o contexto de um país emergente como o Brasil, explorando um período de abrangência mais amplo e profundo em relação aos estudos até então divulgados.

Os resultados empíricos fornecem evidências de que nesse curso há conectividade expressiva das firmas em estudo; porém, as estratégias políticas, seja por doação, seja por integração de membros experimentados em política e/ou por compartilhamento, estão presentes com tipos de aproximações distintas com o governo. Embora muitas ligações com o governo não sejam de fácil visibilidade, determinadas evidências são refletidas no valor das companhias. 
Ao resgatar a hipótese $\mathbf{H 1}$ existe um relacionamento significativo entre as conexões políticas, a partir de laços corporativos, como meio de redução da incerteza, e o desempenho da firma, pode-se supor, em relação ao Q de Tobin, que essa hipótese deve ser confirmada, porque, conforme anteriormente citado. Foram encontradas evidências de que doações a candidatos a presidente e ao governo dos estados-membros e a presença de administradores com antecedentes em política, no legislativo, foram significativas. $\mathrm{O} Q \mathrm{Q}$ de Tobin se mostrou relevante, pois parte expressiva da variabilidade no valor das firmas (Ajd R-squared) é explicada pelo conjunto de mecanismos internos de governança nos dois modelos analisados.

Firmas com vínculos políticos, seja por meio da diretoria ou do conselho, obtêm variação positiva em seu valor, revelando que essas ligações podem representar um fator importante para as políticas corporativas (Bertrand et al., 2006; Goldman et al., 2009; Leuz \& Oberholzer-Gee, 2006). Em oposição, Fan, Wong e Zhang (2007) encontraram baixos desempenhos nas firmas conectadas em relação a outras empresas sem ligação política.

Esses laços, em ambientes regionais com instituições e legislação mais fracas, como, por exemplo, baixa proteção legal, estão mais bem relacionados a melhor desempenho (Li et al., 2008). Isso pode explicar a significância em relação a doações ao cargo de governo.

A segunda hipótese $\mathbf{H} \mathbf{2}$ propõe a seguinte investigação: existe um relacionamento significativo entre as conexões políticas, por intermédio do conjunto de mecanismos internos (conselho de administração e estrutura de propriedade e controle), como meio da redução da incerteza, e o desempenho. As variáveis de propriedade e controle adicionadas ao modelo foram sensíveis somente em relação à categoria de firmas controladas por indivíduos/famílias, $\mathrm{p}<0,001$.

O Q de Tobin é uma medida de valor e contém um componente de mercado, a sua variabilidade está associada a risco avaliado por players de mercado. Dessa forma, não se pode rejeitar a hipótese H2. A minimização de risco importa na redução de incertezas (Barringer \& Harrison, 2000; Bazerman \& Schoorman, 1983). O fato de a empresa ter participação financeira em campanha reporta ao potencial retorno e à influência na distribuição de recursos do governo (Samuels, 2001c). Evidências dessa percepção foram encontradas por Claessens et al. (2008) em investigação sobre acesso preferencial ao crédito financeiro e ao retorno anormal das ações das firmas. Os autores encontraram essa evidência ao analisarem doações à campanha a deputado federal.

Em relação à variável Market-to-book, pode-se aceitar a hipótese H1. Embora a variabilidade das preditoras tenha sido menos influente, comparada ao Q de Tobin, o conjunto de variáveis restou significativo. $\mathrm{O}$ fato de a firma ser doadora, possuir membros do conselho de administração com experiência na administração direta e no legislativo, com práticas de compartilhamento com expolíticos em outros conselhos, apresentou relevância estatística. O Market-to-book, também, associado à incerteza, reúne certo grau de expectativa de retorno futuro (Fan et al., 2007; Jiang, 2008). Já a hipótese $\mathbf{H 2}$ não deve ser aceita, em relação a essa variável, pois nenhuma das categorias de propriedade e controle foi significativa.

Em relação às doações para campanha política em nosso contexto, esses achados contribuem com estudos precedentes de Bandeira-de-Mello e Marcon (2005) e Bandeira-de-Mello et al. (2007), quando analisaram as estratégias políticas das firmas e os efeitos no desempenho, medidos pelo Retorno sobre os Capitais Próprios (ROE). Sob outra base teórica e com conjunto de métricas, essas investigações não encontraram relações significantes entre as firmas doadoras e a diferenciação na performance, o que é confirmado por este estudo quando analisadas outras métricas como variável dependente. As relações firma-governo, por doação, mostram-se duradouras, embora firmas mais ligadas ao governo sejam mais propensas aos efeitos das decisões políticas que daí se originam (Ramalho, 2007). Pode-se deduzir que um período de estabilidade política mais longa pode garantir segurança às firmas ligadas ao governo.

Os dados descritivos do estudo indicam a preponderância de doações a candidatos incumbentes, ou seja, com potencial de serem reeleitos. Mesmo reduzindo a participação de recursos dos candidatos 
vencedores em 2002, foram destinadas, nos três períodos, verbas mais abundantes a candidatos vencedores e houve descentralização das doações de modo a diversificar tais recursos. Os candidatos a presidente, a governador e a deputado federal são os que mais receberam apoio financeiro das firmas. Dos valores doados, os mais beneficiados foram os candidatos ao cargo de deputado estadual/distrital, pois, em relação a 1998, apresentaram maior crescimento de verba recebida.

No contexto americano, Goldman, Rocholl e So (2009), partindo da suposição de que as atividades do conselho de administração não estão restritas ao monitoramento e ao assessoramento à gestão, encontraram evidências de que os membros do board podem adicionar valor por meio de conexões políticas. Salientam a importância da composição com a presença de membros da administração com background em política. Todavia, o interesse da firma em integrar à administração profissionais com experiência em atividade executiva governamental, central ou local pode estar associado a dificuldades financeiras (Claessens et al., 2008; Fan et al., 2007). Embora este estudo não tenha o propósito de investigar esses efeitos, o nível de endividamento pode ser indutor dessas conectividades. O que pode influenciar no valor de mercado da firma.

O pressuposto é o de que a firma sempre vai buscar o meio menos custoso e mais vantajoso para captar recursos (Myers, 1984). Empresas mais endividadas possuem maiores alternativas de acesso a fontes de capitais e, por possuírem mais ativos garantidores desses fundos, têm mais influência no mercado, obtendo custos de transação menos onerosos (Titman \& Wessls 1988).

\section{Conclusões}

Este trabalho, ao se aliar ao corpo teórico que estuda as relações firma-governo, contribui com uma proposta teórica integrativa e desenvolve estratégia de investigação com evidências inovadoras, com amplitude e profundidade metodológica. Demonstra que a exploração de canais de conexão com o ambiente externo possibilita o aumento de valor à firma - medidos pelo Q de Tobin e Market-tobook. Esses indicadores estão associados a risco e incerteza. A relação positiva e significativa demonstra expectativa favorável em relação ao futuro. A literatura aponta que essa aproximação, firma-governo, favorece acesso a financiamentos e a captação de negócios, garantindo o fluxo de recursos essenciais à continuidade e à geração de valor.

Esse papel político é atribuído aos administradores (conselheiros e gestores). Tal atributo do conselho contribui positivamente ao desempenho da firma (Zahra \& Pearce, 1989); por essa razão a participação efetiva do conselho reduz a incerteza e a dependência ambiental. Conselheiros com experiência externa são integrados com a finalidade de cooptar ou parcialmente absorver importantes instituições influentes com as quais as firmas estabelecem relações de interdependência. $\mathrm{O}$ background do conselheiro, o número de interlocks e as características dos membros do conselho são importantes indicadores do grau de orientação da organização. O conhecimento político que esses atores possuem, decorrente de ligações com instituições externas, gera contatos importantes para a firma, produzindo expectativas e reações favoráveis percebidas pelo mercado.

Os dados descritivos sugerem que, em períodos de maior instabilidade política, como nas eleições de 2002, as firmas intensificaram suas estratégias seja por doação e/ou contratação de membros do board com experiência em política.

Deve-se realçar que, não obstante a abrangência metodológica empregada neste estudo, não se pode afirmar com exatidão a relação causa-efeito entre as variáveis testadas. $\mathrm{O}$ fato de a base de dados ser constituída somente por dados secundários, as restrições estabelecidas na constituição da amostra e o período que compreendeu a pesquisa são limitações a serem consideradas. Não foram tratadas no trabalho as firmas com participação minoritária do governo, seja na forma direta ou indireta, o que representa uma limitação. Este escopo de investigação pode ser, em futuros estudos, mais bem 
representado, seja em relação à integração teórica, ao método, à amplitude das variáveis, e, até mesmo, ao período de análise.

Além dessas abordagens, os modelos podem ser testados controlando o nível de risco da firma por meio do modelo de precificação de ativos financeiros (Capital Asset Pricing Model). Por exemplo, firmas com mais risco podem estar mais propensas a se aliarem politicamente.

\section{Artigo recebido em 08.12.2011. Aprovado em 20.08.2012.}

\section{Agradecimentos}

Os autores deste estudo reconhecem e agradecem ao prof. Dr. Wlamir Gonçalves Xavier pelas contribuições prestadas nas análises multivariadas de dados. Destacam que o trabalho foi beneficiado, em muito, com o conhecimento do colega citado.

\section{Referências}

Abor, J. (2005). The effect of capital structure on profitability: an empirical analysis of listed firms in Ghana. The Journal of Risk Finance, 6(5), 438-445. doi: 10.1108/15265940510633505

Agrawal, A., \& Knoeber, C. (1996). Firm performance and mechanisms to control agency problems between managers and shareholders. Journal of Financial and Quantitative Analysis, 31(3), 377-397. doi: $10.2307 / 2331397$

Agrawal, A., \& Knoeber, C. (2001). Do some outside directors play a political role? Journal of Law \& Economics, 44(1), 179-198. doi: 10.1086/320271

Aldrich, H. E., \& Pfeffer, J. (1976). Environments of organizations. Annual Review of Sociology, 2(1), 79-105. doi: 10.1146/annurev.so.02.080176.000455

Bandeira-de-Mello, R., \& Marcon, R. (2005). Unpacking firm effects: modeling political alliances in variance decomposition of firm performance in turbulent environments. Brazilian Administration Review, 2(1), 21-37. doi: 10.1590/S1807-76922005000100003

Bandeira-de-Mello, R., Marcon, R., \& Alberton, A. (2007, August). Stakeholder interaction effects on firm performance in emerging countries: the case of Brazil. Proceedings of the Annual Meeting of the Academy of Management, Philadelphia, PA, EUA.

Barringer, B. R., \& Harrison, J. S. (2000). Walking a tightrope: creating value through interorganizational relationships. Journal of Management, 26(3), 367-403. doi: $10.1177 / 014920630002600302$

Bazerman, M. H., \& Schoorman, F. D. (1983). A limited rationality model of interlocking directorates. Academy of Management Review, 8(2), 206-217. doi: 10.2307/257747

Bertrand, M., Kramarz, F., Schoar, A., \& Thesmar, D. (2006). Politicians, firms and the political business cycle: evidence from France (Unpublished working paper), University of Chicago, Chicago, Illinois, EUA.

Boubakri, N., Cosset, J-C., \& Saffar, W. (2008, October). Political connections and the cost of equity capital. Proceedings of the European Financial Management Symposium on Corporate Governance and Control, Cambridge, Cambridgeshire, United Kingdom. 
Boyd, B. (1990). Corporate linkages and organizational environment: a test of the resource dependence model. Strategic Management Journal, 11(6), 419-430. doi: $10.1002 / \mathrm{smj} .4250110602$

Brennan, N. (2006). Boards of directors and firm performance: is there an expectations gap? Corporate Governance, 14(6), 577-593. doi: 10.1111/j.1467-8683.2006.00534.x

Cho, M-H. (1998). Ownership structure, investment, and the corporate value: an empirical analysis. Journal of Financial Economics, 47(1), 103-121. doi: 10.1016/S0304-405X(97)00039-1

Chung, K. H., \& Pruitt, S. W. (1994). A simple approximation of Tobin's q. The Journal of the Financial Management Association, 23(3), 70-74. doi: 10.2307/3665623

Claessens, S., Feijen, E., \& Laeven, L. (2008). Political connections and preferential access to finance: the role of campaign contributions. Journal of Financial Economics, 88(3), 554-580. doi: 10.1016/j.jfineco.2006.11.003

Daily, C., \& Dalton, D. R. (1994a). Bankruptcy and corporate governance: the impact of board composition and structure. Academy of Management Journal, 37(6), 1603-1617. doi: $10.2307 / 256801$

Daily, C., \& Dalton, D. R. (1994b). Corporate governance and the bankrupt firm: an empirical assessment. Strategic Management Journal, 15(8), 643-654. doi: 10.1002/smj.4250150806

Demsetz, H., \& Lehn, K. (1985). The structure of corporate ownership: causes and consequences. Journal of Politics Economy, 93(6), 1155-1177. doi: 10.1086/261354

Denis, D. K., \& McConnell, J. (2003). International corporate governance. Journal of Financial and Quantitative Analysis, 38(1), 1-36. doi: 10.2307/4126762

Dooley, P. C. (1969). The interlocking directorate. The American Economic Review, 59(3), 314-323.

Faccio, M. (2006). Politically connected firms. The American Economic Review, 96(1), 369-386. doi: $10.1257 / 000282806776157704$

Faccio, M., \& Lang, L. H. P. (2002). The ultimate ownership of western european corporations. Journal of Financial Economics, 65(3), 365-395. doi: 10.1016/S0304-405X(02)00146-0

Fama, E. F. (1980). Agency problems and the theory of the firm. Journal of Political Economy, 88(2), 288-307. doi: $10.1086 / 260866$

Fama, E. F., \& Jensen, M. (1983). Separation of ownership and control. Journal of Law Economics, 26(2), 301-325. doi: 10.1086/467038

Fan, J. H., Wong, T. J., \& Zhang, T. (2007). Politically connected CEOs, corporate governance, and Post-IPI performance of China's newly partially privatized firms. Journal of Financial Economics, 84(5), 330-357. doi: 10.1016/j.jfineco.2006.03.008

Fisman, R. (2001). Estimating the value of political connections. American Economic Review, 4(91), 1095-1102. doi: 10.1257/aer.91.4.1095

Forbes, D. P., \& Milliken, F. J. (1999). Cognition and corporate governance: understanding boards of directors as strategic decision-making groups. Academy of Management Review, 24(3), 489505. doi: 10.5465/AMR.1999.2202133

Goldman, E., Rocholl, J., \& So, J. (2009). Do politically connected boards affect firm value? The Review of Financial Studies, 22(6), 2331-2360. doi: 10.1093/rfs/hhn088

Gujarati, D. (2006). Econometria básica. Rio de Janeiro: Elsevier. 
Hart, O. (1995). Corporate governance: some theory and implications. The Economic Journal, 105(430), 678-689. doi: 10.2307/2235027

Hillman, A. J. (1999). Corporate political strategy formulation: a model of approach, participation, and strategy decisions. Academy of Management Review, 24(4), 825-842. doi: 10.5465/AMR.1999.2553256

Hillman, A. J. (2005). Politicians on the board of directors: do connections affect the bottom line? Journal of Management, 31(3), 464-481. doi: 10.1177/0149206304272187

Hillman, A. J., Cannella, A., Jr., \& Paetzold, R. L. (2000). The resource dependence role of corporate directors: strategic adaptation of board composition in response to environmental change. Journal of Management Studies, 37(2), 235-255. doi: 10.1111/1467-6486.00179

Hillman, A. J., \& Hitt, M. A. (1999). Corporate political strategy formulation: a model of approach, participation, and strategy decisions. Academy of Management Review, 24(4), 825-842. doi: 10.5465/AMR.1999.2553256

Hillman, A. J., \& Keim, G. (1995). International variation in the business-government interface: institutional and organizational considerations. Academy of Management Review, 20(1), 193214. doi: 10.5465/AMR.1995.9503272003

Hillman, A. J., Zardkoohi, A., \& Bierman, L. (1999). Corporate political strategies and firm performance: indications of firm- specific benefits from personal service in the U.S. government. Strategic Management Journal, 20(1), 67-81. doi: 10.1002/(SICI)10970266(199901)

Jiang, C. (2008). The nature of political connections and firma value. In J. J. Choi \& S. Dow (Eds.), Institutional approach to global corporate governance: business systems and beyond. International Finance Review, 9, 461-494. doi: 10.1016/S1569-3767(08)09017-1

Johnson, J., Daily, C. M., \& Ellstrand, A. E. (1996). Boards of directors: a review and research agenda. Journal of Management, 22(3), 409-438. doi: 10.1177/014920639602200303

Johnson, S., \& Mitton, T. (2003). Cronyism and capital controls: evidence from Malaysia. Journal of Financial Economics, 67(2), 351-382. doi: 10.1016/S0304-405X(02)00255-6

Keim, G., \& Baysinger, B. (1988). The efficacy of business political activity: competitive considerations in a principal-agent context. Journal of Management, 14(2), 163-180. doi: $10.1177 / 014920638801400203$

Koenig, T., Gogel, R., \& Sonquist, J. (1979). Models of the significance of interlocking corporate directorates. American Journal of Economics and Sociology, 38(2), 173-186. doi: 10.1111/j.1536-7150.1979.tb02877.x

Lazzarini, S. G. (2011). Capitalismo de laços: os donos do Brasil e suas conexões. Rio de Janeiro: Elsevier.

Lei $n^{o}$ 6.404, de 15 de dezembro de 1976. (1976). Dispõe sobre as Sociedades por Ações. Brasília: Diário Oficial da União.

Lei $n^{\circ}$ 9. 504, de 30 de setembro de 1997. (1997). Lei Eleitoral. Estabelece normas para as eleições. Brasília: Diário Oficial da União.

Leuz, C., \& Oberholzer-Gee, F. (2006). Political relationships, global financing, and corporate transparency: evidence from Indonesia. Journal of Financial Economics, 81(2), 411-439. doi: 10.1016/j.jfineco.2005.06.006 
Li, H., Meng, L., Wang, Q., \& Zhou, L-A. (2008). Political connections, financing and firm performance: evidence from chinese private firms. Journal of Development Economics, 87(2), 283-299. doi: 10.1016/j.jdeveco.2007.03.001

Lynall, M. D., Golden, B. R., \& Hillman, A. J. (2003). Board composition from adolescence to maturity: a multitheoretic view. Academy of Management Review, 28(3), 416-431. doi: 10.5465/AMR.2003.10196743

Mahon, J. F., \& Murray, E. A., Jr. (1981). Strategic planning for regulated companies. Strategic Management Journal, 2(3), 251-262. doi: 10.1002/smj.4250020304

Mizruchi, M. S., \& Stearns, L. B. (1988). A longitudinal study of the formation of interlocking directorates. Administrative Science Quarterly, 33(2), 194-210. doi: 10.2307/2393055

Myers, S. C. (1984). The capital structure puzzle. Journal of Finance, 39(3), 575-592. doi: 10.1111/j.1540-6261.1984.tb03646.x

Myers, S. C., \& Majluf, N. S. (1984). Corporate financing and investment decisions when firms have information that investors do not have. Journal of Financial Economics, 13(2), 187-221. doi: $10.1016 / 0304-405 X(84) 90023-0$

Odegaard, B. A., \& Bøhren, Ø. (2004). Governance and performance revisited (Finance Working Paper $N^{\circ}$ 28/2003; EFA 2003 Annual Conference Paper $N^{\circ}$ 252). Social Science Research Networks. Recuperado de http://papers.ssrn.com/sol3/ papers.cfm? abstract_id=423461

Okhmatovskiy, I. (2010). Performance implications of ties to the government and SOEs: a political embeddedness perspective. Journal of management studies, 47(6), 1020-1047. doi: 10.1111/j.1467-6486.2009.00881.x

Pearce, J. A., II (1983). The relationship of internal versus external orientations to financial measures of strategic performance. Strategic Management Journal, 4(4), 297-306. doi: $10.1002 / \mathrm{smj} .4250040402$

Pearce, J. A., II, \& Zahra, S. A. (1992). Board composition from a strategic contingency perspective. Journal of Management Studies, 29(4), 411-438. doi: 10.1111/j.1467-6486.1992.tb00672.x

Petersen, M. A. (2009). Estimating standard errors cin finance panel data sets: comparing approaches. The Review of Financial Studies, 22(1), 435-480. doi: 10.1093/rfs/hhn053

Pfeffer, J. (1972). Size and composition of corporate boards of directors: the organization and its environment. Administrative Science Quarterly, 17(2), 218-229.

Pfeffer, J., \& Salancik, G. R. (2003). The external control of organizations: a resource dependence perspective. Stanford, CA: Stanford Business Books.

Pittman, R. (1977). Market structure and campaign contributions. Public Choise, 31(1), 37-52. doi: 10.1007/BF01718973

Ramalho, R. (2007). The persistence of corruption: evidence from the 1992 presidential impeachment in Brazil. The World Bank. Recuperado de http://corruptionresearchnetwork.org/resources/articles/the-persistence-of-corruption-evidencefrom-the-1992-presidential-impeachment-in-brazil/?searchterm=ramalho

Rediker, K. J., \& Seth, A. (1995). Boards of directors and substitution effects of alternative governance mechanisms. Strategic Management Journal, 16(2), 85-99. doi: $10.1002 /$ smj. 4250160202 
Samuels, D. J. (2001a). Incumbents and challengers on a level playing field: assessing the impact of campaign finance in Brazil. The Journal of Politics, 63(2), 569-584. doi: 10.1111/00223816.00079

Samuels, D. J. (2001c). Does money matter? credible commitments and campaign finance in new democracies: theory and evidence from Brazil. Comparative Politics, 34(1), $23-42$.

Samuels, D. J. (2001b). Money, elections, and democracy in Brazil. Latin American Politics and Society, 43(2), 27-48. doi: 10.2307/3176970

Schoorman, F. D., Bazerman, M. H., \& Atkin, R. S. (1981). Interlocking directorates: a strategy for reducing environmental uncertainty. Academy of Management Review, 6(2), 243-251. doi: 10.5465/AMR.1981.4287813

Schuler, D. A., Rehbein, K., \& Cramer, R. D. (2002). Pursuing strategic advantage through political means: a multivariate approach. Academy of Management Journal, 45(4), 659-672. doi: $10.2307 / 3069303$

Shleifer, A., \& Vishny, R. W. (1986). Large shareholders and corporate control. Journal of Political Economy, 94(3), 461-488. doi: 10.1086/261385

Shleifer, A., \& Vishny, R. W. (1997). A survey of corporate governance. The Journal of Finance, 52(2), 737-783. doi: 10.1111/j.1540-6261.1997.tb04820.x

Silva, A. L. C., \& Graminho, F. M. (2005). Campaign finance and corporate governance: the case of Brazil. Corporate Ownership \& Control, 3(2), 125-136.

Thomsen, S., \& Pedersen, T. (2000). Ownership structure and economic performance in the largest european companies. Strategic Management Journal, 21(6), 689-705. doi: 10.1002/(SICI)10970266(200006)21:6<689::AID-SMJ115>3.0.CO;2-Y

Titman, S., \& Wessels, R. (1988). The determinants of capital structure choice. Journal of Finance, 43(1), 1-19. doi: 10.1111/j.1540-6261.1988.tb02585.x

Venkatraman, N., \& Ramanujam, V. (1986). Measurement of business performance in strategy research: a comparison of approaches. Academy of Management Review, 11(4), 801-814. doi: 10.5465/AMR.1986.4283976

Westphal, J. D. (1999). Collaboration in the boardroom: behavioral and performance consequences of CEO-board social ties. Academy of Management Journal, 42(1), 7-24. doi: 10.2307/256871

Wooldridge, J. (2003). Econometric analysis of cross section and panel data. Cambridge, MA: MIT Press.

Yermack, D. (1996). Higher market valuation of companies with a small board of directors. Journal of Financial Economics, 40(2), 185-211. doi: 10.1016/0304-405X(95)00844-5

Yoffie, D. B. (1988). How an industry builds political advantage. Harvard Business Review, 66(3), 8289. doi: $10.1225 / 88314$

Zahra, S. A., \& Pearce, J. A. II (1989). Boards of directors and corporate financial performance: a review and integrative model. Journal of Management, 15(2), 291-334. doi: $10.1177 / 014920638901500208$ 